\title{
Mesencephalic and third ventricle cysts: diagnosis and management in four cases
}

\author{
V Th Ramaekers, J Reul, V Siller, A Thron
}

\begin{abstract}
Four infants with obstructive hydrocephalus caused by space occupying third ventricle and mesencephalic cysts are reported. Despite immediate shunt insertion in all patients, there was either lack of clinical improvement or late onset of clinical deterioration. Neuroimaging (CT, MRI, and ventriculography) diagnosed the presence of non-communicating midline outpouchings of the CSF pathways causing obstruction of aqueductal CSF flow and brainstem signs. The cysts were of different origin. In one patient it was caused by a previous thalamic haemorrhage, in another patient by neonatal Escherichia coli meningoventriculitis. In two cases with obstructive hydrocephalus at birth, the aetiology is unclear. Direct puncture and drainage of the cysts led to clinical improvement. The cysts were poorly visualised on CT and could be misinterpreted as an enlarged third ventricle, simulating congenital aqueduct stenosis. Careful neuroradiological investigation is necessary to establish an accurate diagnosis and neurosurgical management. In such cases with hydrocephalus and persisting ventricular enlargement despite shunting, CT ventriculography is a useful tool.
\end{abstract}

(F Neurol Neurosurg Psychiatry 1994;57:1216-1220)

Department of Paediatrics, University of Aachen, Pauwelsstrasse 30, D-52057 Aachen, Germany V Th Ramaekers

Department of Neuroradiology, University of Aachen, Pauwelsstrasse 30, D52057, Aachen, Germany J Reul

A Thron

Bethlehem Krankenhaus, Stolberg, Germany V Siller

Correspondence to: Dr V Th Ramaekers, Department of Pediatrics, Medizinische Einrichtungen der RWTH Aachen, Pauwelsstrasse 30, D-52057 Aachen, Germany.

Received 1 September 1993 and in revised form 25 March 1994. Accepted for publication 3 May 1994
Obstructive hydrocephalus at birth associated with dilatation of the third and lateral ventricles most commonly results from aqueduct stenosis. In most cases the stenosis is sporadic and non-genetic, but rare $\mathrm{X}$ linked cases occur, often associated with adducted thumbs and with a poor prognosis. ${ }^{1-3}$ Prenatal infection (toxoplasmosis, cytomegalovirus), postnatally acquired meningitic ependymitis, or intracranial haemorrhage can also cause aqueduct stenosis with obstructive hydrocephalus, but the history and the presence of signs such as periventricular calcifications or chorioretinitis can help to differentiate between the various conditions. ${ }^{3}$ Establishing the correct diagnosis in an affected newborn is important for reasons of genetic advice and management. The presence of gross enlargement of the third and lateral ventricles on CT, however, can sometimes obscure the exact underlying aetiology for obstruction of CSF flow. Particularly, diagnostic difficulties can arise in the differentiation between aqueduct stenosis, the presence of cysts within the third ventricle, and small cysts located in the pontomesencephalic region with compression of the aqueduct canal. ${ }^{4-6}$ Even suprasellar subarachnoid outpouchings can cause compression on the floor of the third ventricle or aqueduct thereby disturbing normal CSF passage from the third ventricle towards the aqueductal canal. $^{6}$

The present report highlights the clinical and neuroradiological findings as well as the neurosurgical management in four cases with congenital and postnatally acquired obstructive hydrocephalus due to blockage of CSF flow by midline cysts or outpouchings of suprasellar subarachnoid spaces.

\section{Case reports}

CASE 1

After a normal pregnancy, the first daughter of healthy parents had to be delivered by caesarian section because of heart beat deceleration and craniopelvic disproportion. The neonatal period was uneventful. At the age of nine weeks she was referred to us because of accelerated head growth, poor suckling, and generalised hypotonia. Her head circumference was enlarged $(42 \mathrm{~cm}$; $>$ P97) and the anterior fontanel was full and tense with separated cranial sutures. On clinical examination she had a high pitched cry, poor suckling and swallowing, generalised hypotonia with normal reflexes, and failure of visual fixation with disconjugate and roving eye movements. Obstructive hydrocephalus was confirmed by cranial ultrasonography and CT, which showed pronounced dilatation of the third and lateral ventricles as well as an infratentorial midline cyst (fig 1A). After insertion of a ventriculoperitoneal shunt into the right frontal horn, the size of the ventricles diminished but clinical signs of hypotonia, poor feeding, and intermittent disconjugate upward eye deviation persisted. After one week follow up CT and MRI (fig $1 \mathrm{C}$ and $\mathrm{D}$ ) showed persistance of a CSF isodense midline cyst with pontomesencephalic location. The expanding cyst caused distortion of brainstem structures and compression of the aqueduct. Ventriculography (fig 1B) showed the absence of free communication between the ventricular system and the pontomesencephalic cyst. The life threatening brainstem compression by this expanding cystic lesion urged further neurosurgical intervention. A communication between the cyst and right lateral ventricle was established by 


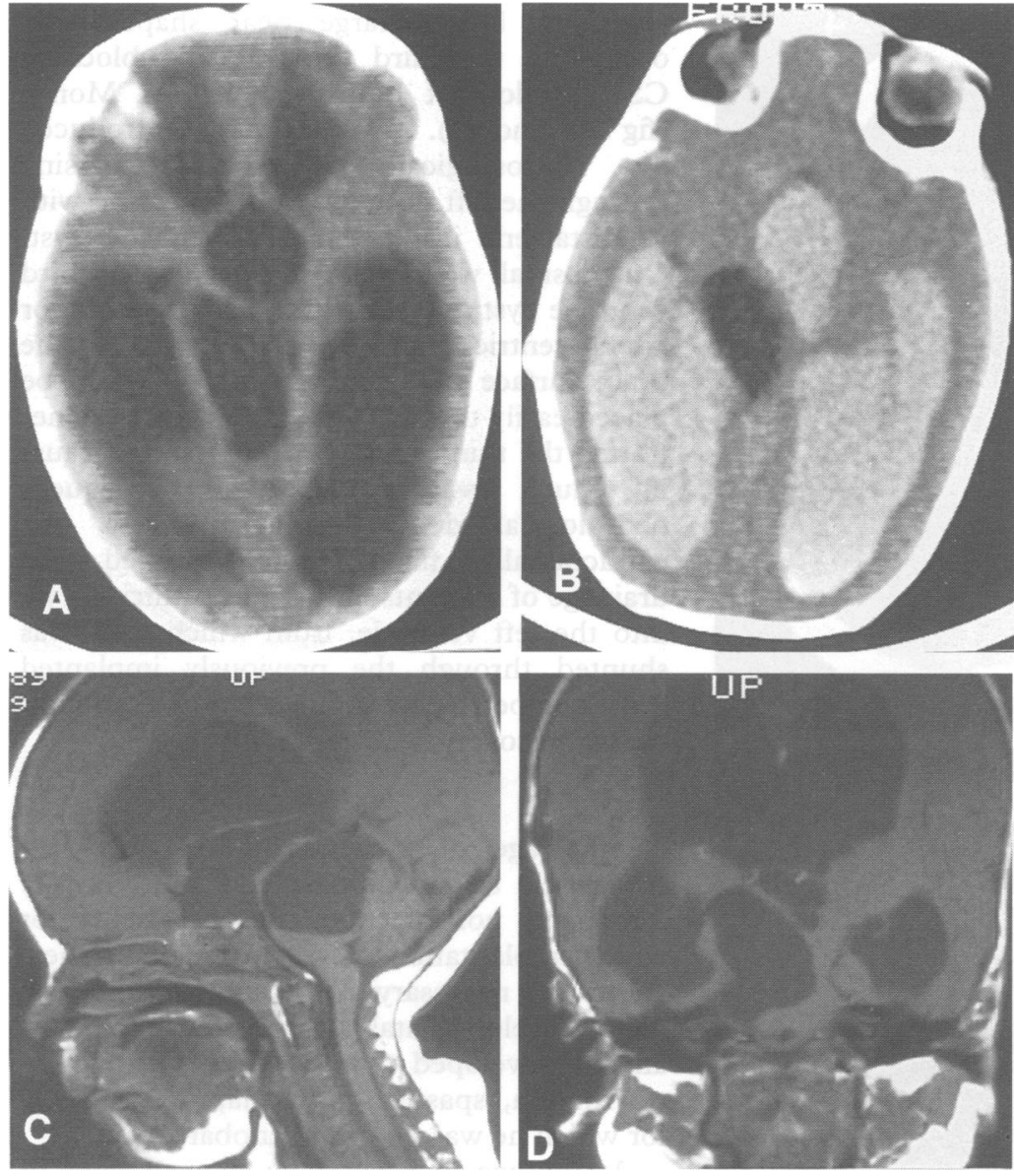

Figure 1 (case 1) (A) Obstructive hydrocephalus with pronounced dilatation of the supratentorial ventricles and an infratentorial cystic lesion. (B) Ventriculography clearly shows the absence of a free communication between the ventricles and the pontomesencephalic cyst. ( $C$ and D) T1 weighted MRI showing a large mesencephalic cyst compressing the aqueduct. A communication to the ventricular system cannot be excluded.

implantation of a cystoventricular shunt. Postoperatively, the patient's condition improved rapidly and one month later cranial ultrasound showed reduction in size of the pontomesencephalic cyst. Four months later the size of the cyst and the third and lateral ventricles had reduced considerably. The feeding difficulties and roving eye movements disappeared and she started to fix and follow visual lures. At the age of 7 months her motor development was still delayed with signs of left sided convergent squint and hemiparesis.

CASE 2

After delivery by caesarian section, the patient needed immediate resuscitation because of severe postnatal apnoea and bradycardia attributed to severe hydrocephalus. His head circumference was very enlarged $(47 \mathrm{~cm})$. Height $(52 \mathrm{~cm})$ and weight $(3860 \mathrm{~g})$ were normal. Lumbar puncture pneumoencephalography (in 1974) showed normal air filling of the cervical spinal canal and suboccipital and basal cisterns but no filling of the ventricular system. Transfontanel puncture showed a considerably enlarged lateral and third ventricular system but no visualisation of the aqueduct of Sylvius. The presumptive diagnosis was consistent with congenital aqueduct stenosis after exclusion of chorioretinitis and TORCH or Listeria infection and a ventriculoatrial shunt was implanted. Clinical follow up showed pronounced delay of mental and motor milestones. By the age of 3 years the patient could walk independently but his gait was ataxic with signs of spastic paraplegia. His vision was poor with acuity of $2 / 10$ and funduscopy showed non-progressive bilateral optic atrophy. He developed peculiar eye movements consisting of intermittent upward eye deviation associated with rhythmic horizontal head bobbing. ${ }^{7}$ From 4 years left sided hemiconvulsions occurred and these were treated with primidone. At the age of 6 years shunt revision was necessary because of acute hydrocephalus (fig 2A and B). Follow up CT at 15 years because of sudden clinical deterioration with acute pressure signs indicated the presence of a large third ventricle cyst blocking the foramina of Monro (fig 2C). Neurosurgical intervention alleviated the acute pressure signs and was followed by prompt clinical recovery. The cyst was punctured and drained by a separate drain that was connected to the existing ventricular shunt by a Y connection (fig 2D). Postoperative CT now clearly illustrated callosal agenesis and evidence for pachygyria particularly in the parieto-occipital cortex. The boy still has severe mental retardation and recurrent episodes of abnormal upward eye deviation with preserved consciousness. Although his gait has improved, pyramidal signs in the lower limbs and a discrete ataxia still persist.

CASE 3

After a gestational period of 35 weeks complicated by eclampsia, this patient was delivered by caesarian section because of fetal distress. The Apgar score was 8-9-10 (umbilical artery $\mathrm{pH} 7 \cdot 26$ ). His birth weight was $1900 \mathrm{~g}$, length $42 \mathrm{~cm}$, and head circumference $30.5 \mathrm{~cm}$. Inadequate respiration prompted treatment with oxygen during the first hours after birth, on which he recovered quickly. One $\mathrm{mg}$ vitamin $\mathrm{K}$ was given intramuscularly. After an uneventful neonatal period during the first 20 days, sudden clinical deterioration with seizures and a fall in haemoglobin content occurred as a consequence of a right sided intrathalamic haemorrhage with intraventricular extension (fig 3A). After development of posthaemorrhagic hydrocephalus (fig 3B) an intraventricular Rickham reservoir was inserted for regular ventricular taps.

Follow up examination by cranial ultrasound and CT two weeks later showed complete resolution of the intrathalamic bleeding site and after three months, transformation into a low density area. The borders of the lesion did not show contrast enhancement. Despite regular taps through the Rickham reservoir, accelerating head growth and raised intracranial pressure necessitated insertion of a ventriculoperitoneal shunt at the age of 4 months. At the age of 6 months infantile 

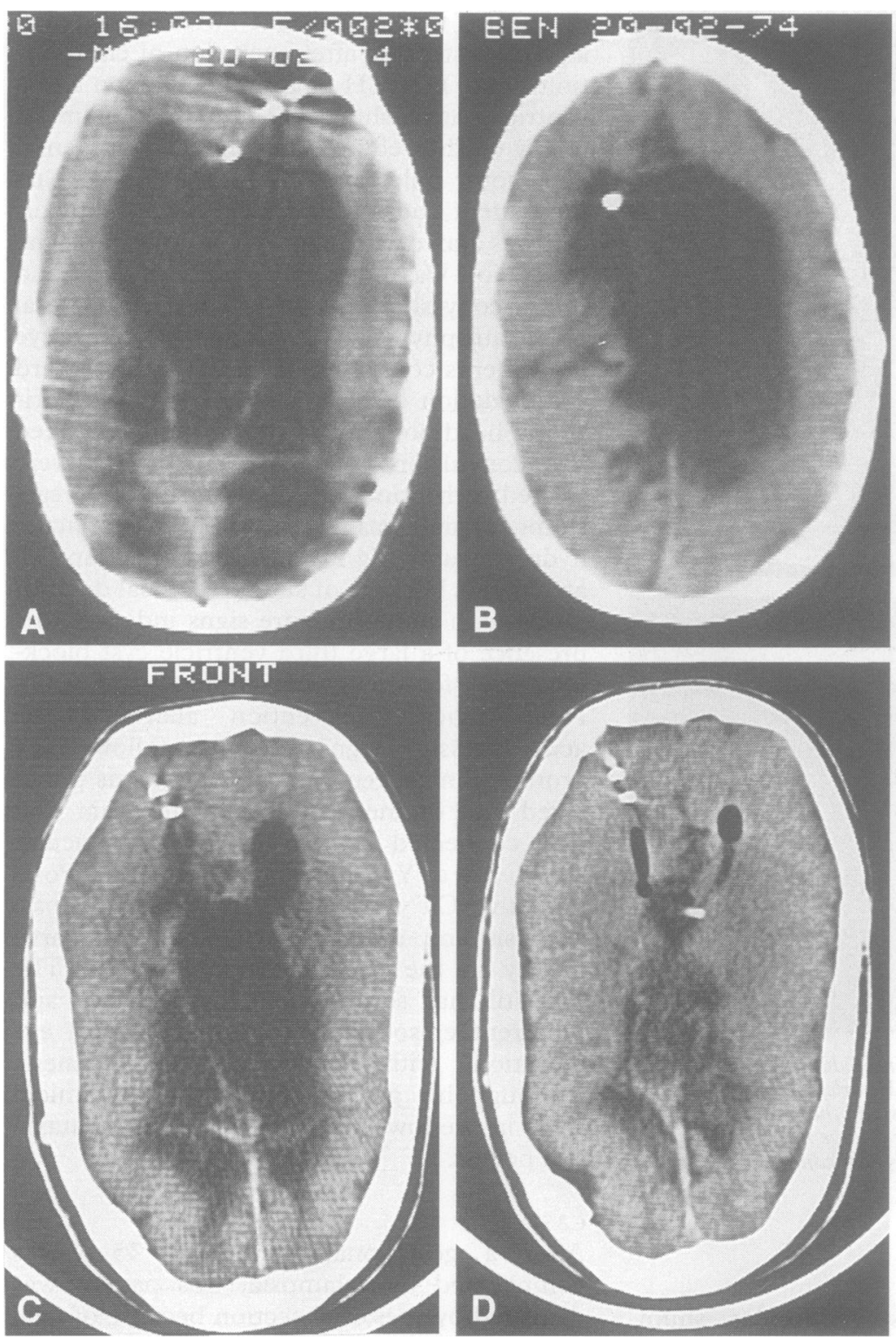

Figure 2 (case 2) (A) Pronounced dilatation of the ventricular system despite a frontal shunt system. The large midline cyst is not identifiable. (B) After shunt revision with a shunt system placed in the left frontal horn. Ventricles are still asymmetric, but reduced in size. (C and D) Nine years later CT shows a small ventricular system without signs of shunt dysfunction and a large midline cyst. Placement of a second shunt system into the cyst resulted in the collapse of the cyst, which did not communicate with the ventricles.

spasms had to be treated several times with $\mathrm{ACTH}$ and phenobarbitone until the age of 1 year. Follow up examination showed gross developmental delay with a right sided squint and signs of spastic diplegia. At the age of 2 years a febrile gastrointestinal infection with Salmonella typhimurium was accompanied by generalised seizures 30 minutes in duration. After six days he was transferred to our hospital because of insufficient postictal recovery, intermittent periods of somnolence, and recurrent vomiting. An EEG showed diffuse slowing with delta activity particularly over the right hemisphere. Funduscopic examination excluded papilloedema but showed discrete pallor of the left optic disc. On CT the third ventricle appeared grossly enlarged compared with its size on previous CT. Contrast injection into the ventriculoperitoneal shunt showed normal contrast filling of both lateral ventricles but a large pear shaped cyst occupying the third ventricle and blocking CSF outflow at both foramina of Monro (fig 3C and D). A catheter was introduced by neurosurgical intervention, passing through the left occipital horn and lying with its distal end inside the third ventricle cyst. Only a small wall separated the enlarged third ventricle cyst and the distended left posterior lateral ventricle horn, which was close to the brain surface (fig 3D). So a drain could be placed easily through the occipital horn penetrating the thin separating wall without causing much tissue damage and consequent neurological deficits. Postoperatively, the extracranially fixed shunt enabled the drainage of contents of the third ventricle cyst into the left ventricle, from which CSF was shunted through the previously implanted ventriculoperitoneal shunt. There was prompt clinical recovery.

CASE 4

At the age of 2 weeks the patient had Escherichia coli meningitis and subsequently developed postinfectious hydrocephalus for which implantation of a ventriculoperitoneal shunt was necessary at the age of 3 months. Neurodevelopmental progress was delayed and he developed cerebral palsy with mental retardation, spastic tetraplegia, and epilepsy for which he was put on phenobarbitone.

At the age of 8 years he showed signs of raised intracranial pressure due to acute hydrocephalus caused by insufficient ventriculoperitoneal drainage. Preoperative CT showed dilated lateral ventricles and for the first time a non-communicating cystic lesion at the level of the third ventricle was suspected (fig $4 \mathrm{~A}$ ).

From the age of 8 years he complained several times of headaches and nausea, but there were no signs of raised intracranial pressure. From the age of 13 years these symptoms relapsed more frequently, and subsided spontaneously. A CT at that age showed a large midline cyst bulging into the third ventricle (fig 4B). At 15 years he suddenly developed nausea, vomiting, and headaches and became somnolent. A new shunt system was implanted but he continued to have headaches. Ventriculography and CT confirmed a local expanding cyst within the third ventricle, which had no free communication with the ventricular system (fig 4C and D).

Several neurosurgical procedures were needed to reestablish communication between the cyst and lateral ventricles. Two shunt systems have been inserted draining the left and right lateral ventricles. To establish a free communication between the third ventricle cyst and the lateral ventricles, a stereotactic guided fenestration of the cyst walls was performed. The symptoms and signs of raised intracranial and local pressure had disappeared at postoperative follow up.

\section{Discussion}

Demonstration by CT of obstructive hydrocephalus with dilatation of the third and 

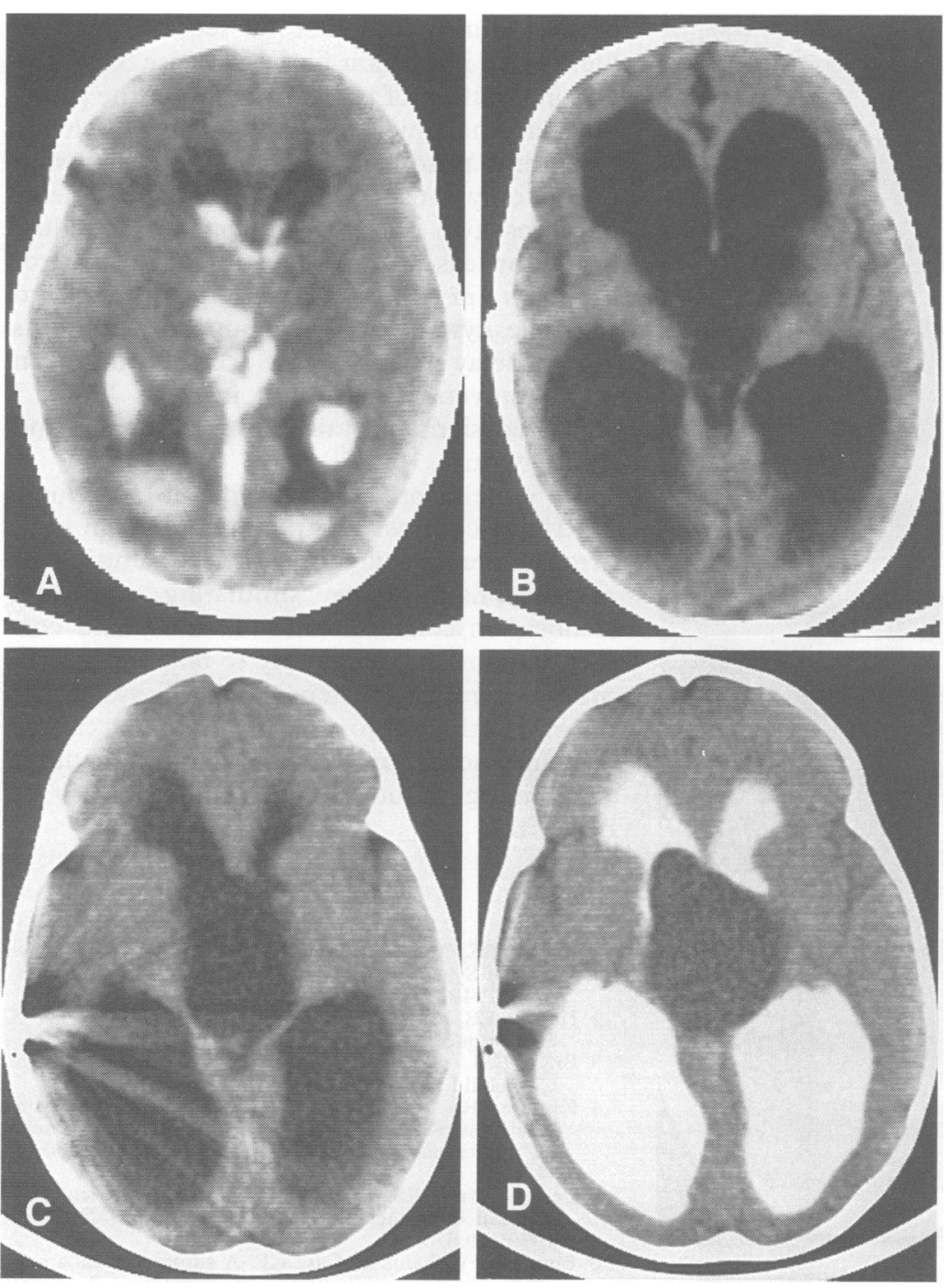

Figure 3 (case 3) (A) Right sided thalamic bleeding with intraventricular extension. (B) Resorption of the haemorrhage was followed by pronounced dilatation of the ventricular system requiring neurosurgical shunting. ( $C$ and $D)$ After insertion of a shunt the enlargement of the third ventricle still persists and ventriculography shows a large noncommunicating cyst blocking CSF outflow at the foramina of Monro.

lateral ventricles in the presence of a normal sized fourth ventricle indicates obstruction of CSF flow through the aqueduct of Sylvius. In most congenital cases the underlying cause is unknown and presents a sporadic non-genetic occurrence, but in a few patients a genetically $\mathrm{X}$ linked aqueductal stenosis is present. ${ }^{1-3}$ In some cases, prenatal infections due to cytomegalovirus and toxoplasmosis or postnatally acquired meningitic ependymitis can also stenose, distort, or obstruct the aqueductal canal. ${ }^{3}$ Pitfalls in the establishment of a correct diagnosis can occur, however; a suprasellar subarachnoid cyst or craniopharyngeoma may enlarge and extend into the floor of the third ventricle to obstruct CSF flow at the foramina of Monro or the exit of the third ventricle. The resulting appearance on CT of obstructive hydrocephalus may sometimes simulate stenosis of the aqueduct. ${ }^{58}$ In such cases Leo et al have advocated the use of contrast ventriculography because the contrast medium will not enter the cyst but will enter a dilated third ventricle in a patient with stenosis of the aqueduct. ${ }^{5}$ Kishore et al have also pointed out the limitation of diagnosis of intracranial midline cysts (craniopharyngeoma, epidermoid cyst) by CT, particularly those midline cystic lesions with thin noncontrast enhancing walls containing CSF isodense material. ${ }^{4} \mathrm{~A}$ further limitation of $\mathrm{CT}$ in correct diagnosis of these lesions is related to variations in the anatomy at the suprasellar region and the skull base. ${ }^{9}$ Binitie et al documented a child with hydrocephalus caused by a suprasellar subarachnoid outpouching that distended and filled slowly by a valve mechanism. This subsequently invaginated the floor of the third ventricle, and eventually evolved into a cystic lesion occupying space at the site of the third ventricle and obstructing normal CSF flow. ${ }^{6}$

Therefore, in selected cases a strategy should be chosen for additional neuroimaging by MRI, ventriculography, or CT metrizamide cisternography. ${ }^{4-6}$

As cases 1 and 3 have shown, the persistence of brainstem signs after shunted hydrocephalus (abnormal eye movements, somnolence, seizures, hypotonia, poor suckling, and persistent vomiting) should raise suspicion as to the presence of an underlying third ventricle or brainstem cyst with aqueduct compression and should lead to further neuroradiological investigations. ${ }^{4-10}$ Among suspected cases follow up CT after shunt implantation should be performed early because visualisation of an enlargement of midline CSF pathways can confirm the suspicion of the presence of a cyst. Early diagnosis of mesencephalic cysts with obstructive hydrocephalus is important because surgical intervention with drainage can prevent further expansion of pontomesencephalic cysts and avoid life threatening and irreversible damage to vital brainstem structures. As cases 1, 3, and 4 have illustrated, MRI or contrast ventriculography through a previously implanted ventriculoperitoneal shunt can visualise and outline the cyst margins and determine whether it is communicating with the ventricular system or not.

The underlying aetiology of the mesencephalic cyst in cases 1 and 2 remains unclear, but an arteriovenous malformation or tumour was ruled out. This was supported by follow up neuroimaging that did not show any change or contrast enhancement by the cyst margins. In cases 2 and 4 the suprasellar subarachnoid pouch invaginating the floor of the third ventricle and eventually causing obstructive hydrocephalus ${ }^{6}$ could be the possible pathogenetic factor. A lumbar cisternography may be the best way to confirm this, but it was not done in our patients. If a pouch and not a cyst, however, was the underlying aetiology, the neurosurgical treatment would be similar. Moreover, the development of Escherichia coli meningitic ependymitis during the neonatal period in case 4 was believed to be responsible for the development of a postinfectious cyst within the ventricular system. In case 3 the unexpected clinical deterioration due to unilateral intrathalamic bleeding with intraventricular extension at the age of 20 days could 

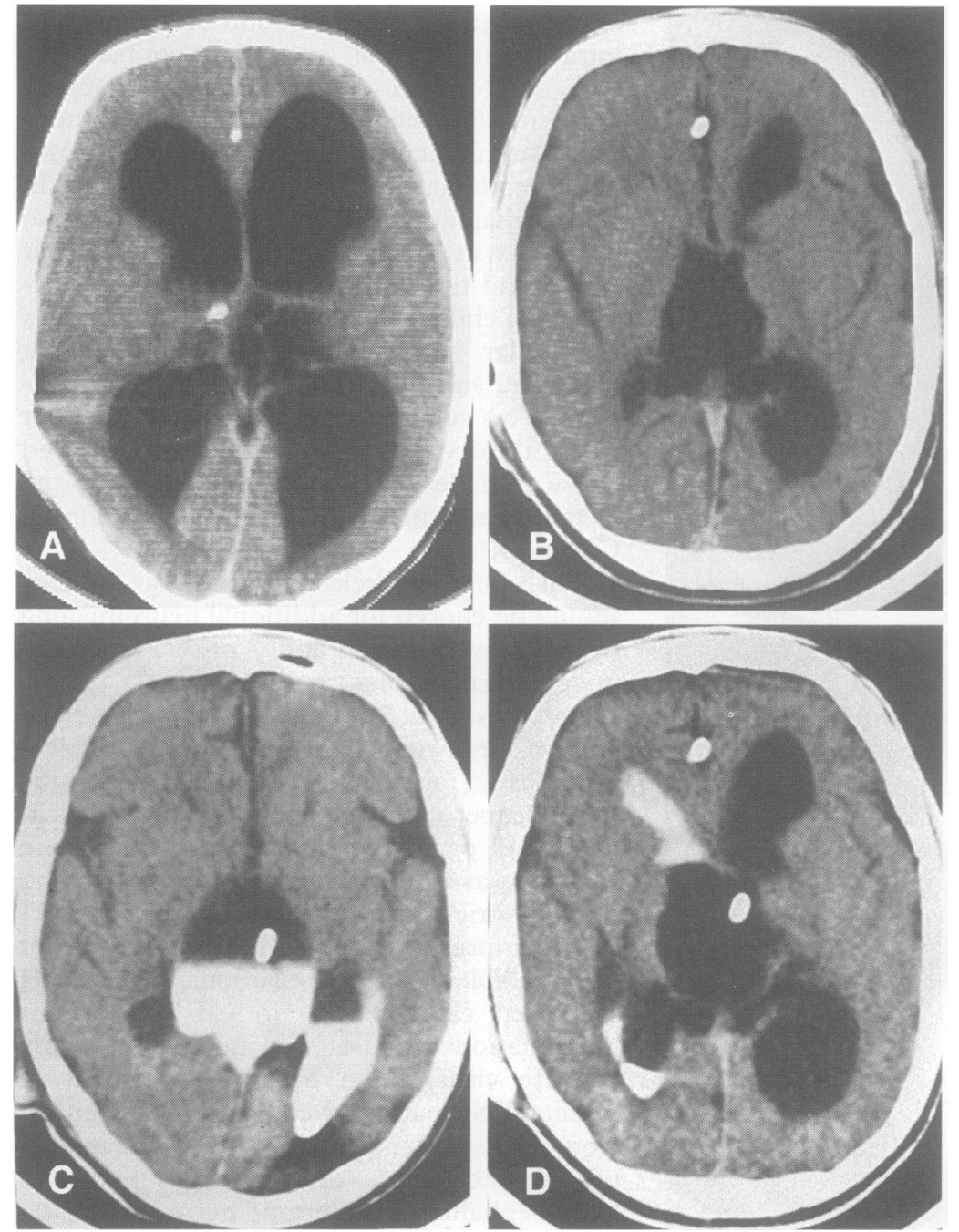

Figure 4 (case 4) (A) CT shows a cystic formation close to the third ventricle without communication and hydrocephalus internus despite shunting. (B) Control scans show a large midline cyst separated from the third ventricle, producing occlusive hydrocephalus. (C) Filling of the cyst with contrast medium shows absence of communication to the ventricles. (D) No opacification of the cyst is visible at ventriculography.

not be explained by an arteriovenous malformation. Unilateral thalamic haemorrhage with intraventricular extension has been reported in full term infants who were initially well but presented with sudden clinical deteri- oration during the first or second week of life. ${ }^{11}{ }^{12}$ An important finding in case 3 was the documentation of transformation of the previous intrathalamic bleeding site into a slowly expanding third ventricle cyst. The expansion of this only caused clinical signs after a delay of almost two years. A similar course is illustrated by case 4 with postinfectious hydrocephalus, in whom expansion of the third ventricle cyst caused pronounced symptoms after a delay of 14 years. Successful insertion of a cystoventricular shunt in cases 1 and 3 alleviated signs of brainstem dysfunction and local pressure.

In conclusion, the advent of additional MRI and contrast ventriculography can confirm the correct diagnosis of third ventricle or mesencephalic cysts simulating aqueduct stenosis on conventional CT. Early and correct diagnosis is mandatory to take the appropriate therapeutic steps. Regardless of the aetiology, the neurosurgical treatment can be difficult and in some cases several procedures (combining shunts and fenestration) may be necessary.

1 Bickers DS, Adams RD. Hereditary stenosis of the aqueduct of Sylvius as a cause of congenital hydrocephalus. duct of Sylvius as

2 Holmes LB, Nash A, Zurhein GM, Levin M, Opitz JM. X linked aqueductal stenosis. Pediatrics 1973;51:697-704.

3 Brett EM. Paediatric neurology. 2nd ed. Edinburgh: Churchill Livingstone 1991: 467-8.

4 Kishore PRS, Krishna Rao CVG, Williams JP, Vines FS. The limitation of computerized tomographic diagnosis of intracranial midline cysts. Surg Neurol 1980;14: 417-31.

5 Leo JS, Pinto RS, Hulvat GF, Epstein F, Kricheff II Computed tomography of arachnoid cysts. Radiology 1979;130:675-80

6 Binitie O, Williams B. Case CP. A suprasellar subarachnoid pouch; aetiological considerations. $\mathcal{f}$ Neurol Neurosurg Psychiatry 1984;47:1066-74.

7 Nellhaus G. The bobble-head doll syndrome: a "tic" with a neuropathologic basis. Pediatrics 1967;40:250-3.

8 Lee BCP. Intracranial cysts. Radiology 1979;130:667-74.

9 Wiggli W, Benz FU. Normal computed tomography anatomy of the suprasellar subarachnoid space. anatomy of the suprasell

10 Fenton T, Seton C, Hall D, Kendall B. Intermittent disconjugate eye movements - a sign of raised intracranial pressure with brain stem compression. Neuropediatrics 1989;20:30-2.

11 Roland EH, Flodmark O, Hill A. Thalamic haemorrhage with intraventricular haemorrhage in the full-term newborn. Pediatrics 1990;85:737-42.

12 Trounce JQ, Fawer CI, Punt J, Dodd KL, Fielder AR, Levene MI. Primary thalamic haemorrhage in the newborn: a new clinical entity. Lancet 1985;i:190-2. 